\title{
Using Factor Analysis to Study the Critical Success Factors of Agile Software Development
}

\author{
Abdullah Aldahmash*, Andrew Gravell, Yvonne Howard \\ School of Electronics and Computer Science, University of Southampton, Southampton, UK. \\ * Corresponding author. Tel.: +4407414140364; email: a.aldahmash@soton.ac.uk \\ Manuscript submitted August 25, 2017; accepted October 8, 2017. \\ doi: 10.17706/jsw.12.12.957-963
}

\begin{abstract}
This paper aims to study the critical success factors of agile software development projects. Following a systematic review of the literature, eight success factors were selected as critical success factors of agile software development. A web based survey was designed to collect data about agile practitioners' opinions concerning these success factors. Principal component analysis is conducted to identify the latent dimensions and how the selected success factors of agile development are related. This analysis of the eight success factors resulted in clustering them into two components. The first component labelled as Organizational and People Aspects which consist of five items which are: organizational culture, customer involvement, top management support, team capability and training, and communication. The second component labelled as Technical and Project Management Aspects which consist of three items which are: project management process, delivery strategy, and agile software techniques. The clustering of the success factors of agile projects which presented in this paper will help agile practitioners to understand how these success factors are related to each other, and may help in planning or improving agile training programmes.
\end{abstract}

Keywords: Agile success, factor analysis, factor extraction, software development, success factors.

\section{Introduction}

Agile software development techniques and methods have been developing since the release of the agile manifesto [1]. In 2001, a group of software practitioners introduced four values of agile which are: individuals and interactions over processes and tools, working software over comprehensive documentation, customer collaboration over contract negotiation, and responding to change over following a plan. Agile principles shift the attention from following the plan to adding value by emphasizing on delivering the working software frequently in short timespans [2]. Agile development principles encourage the team to deliver to the customer as early as possible and then receive feedbacks early. So as to simplify the changes and to improve the quality aiming to achieve the customer's satisfaction [3].

The last few decades have produced a great deal of research focused on identifying the factors that influence the success of software development. According to Leidecker \& Bruno [4], the critical success factors are those factors, conditions, and attributes that, when rightly addressed, managed have a huge influence on the success of the project.

While the agile development spreads, the question of how to achieve the success in implementing and adopting agile methods raised. Many studies have investigated the critical success factors (CSFs) of agile development projects. For instance, Chow \& Cao [5] studied the critical success factors and identified a set of success factors for agile software development.

In this paper, the relationships between these success factors will be investigated. Using factor analysis, a 
sort of grouping or clustering the CSFs will be beneficial in simplifying how to present and understand the success factors of agile development projects. The data were collected by a web-based survey and the sample size was 131 responses. The factor analysis resulted with grouping the eight success factors into two components. The first component is related to the people and organizational aspects. The second component is related to the technical and project management aspects.

This paper is organized as follows: Section 1 is an introduction, and Section 2 is a background. Section 3 provides an overview of the data collection and preparation. Section 4 presents the factor extraction, Section 5 shows the factor interpretation and labelling. Section 6 is the discussion and Section 7 provides conclusion and suggestions for future work.

\section{Background}

Agile software development has many characteristics, all of which are identified in the literature. According to Dyba \& Dingsoyr [6] the key characteristics of agile development are collaboration and communication, embracing conflicts, and supporting creativity and innovation; managers are only facilitators, and the design and implementation processes are iterative and indivisible. Vijayasarathy \& Turk [7] conducted a survey which revealed that the use of agile development will lead to the ability to meet customer needs and to deliver quality software. Other identified benefits of using agile development include the increasing of flexibility in development, as well as low development costs, more frequent working software delivery, and more reusable code. Since many facets of software development exist in a rather complex domain, agile development could be seen as a technique with which to address this complexity [8]. According to the State of Agile Survey 2016 [9], the top three reasons for adopting agile were to accelerate product delivery, enhance the ability to manage changing priorities, and increase productivity.

Most of the agile practices focus on customer involvement and developers empowerment. Agile practices also encourage changes and improve the responses to those changes. Therefore, adopting agile requires a culture change and a new method of communication and coordination. The traditional rigor control mechanisms within the organizations, such as the command/control management approach, must also be changed [10].

Turk, France, \& Rumpe [11] pointed out that many of the agile principles, ideas and approaches are built on assumptions which are probably not suitable for all software development projects. Thus, when an organization finds out that some of the agile principles will not fit its case, these principles should be partially adapted, or simply ignored altogether. The worst scenario for an organization is to try to enforce all of the agile principles. The organization must be sure of those assumptions before going with agility. If this is not done, the agility will probably provide less value for the organization.

Pikkarainen et al. [12] studied the case of successful agile software development projects in three software companies in Finland. The study revealed the importance of management support and belief in agile. The study also found that organizations adopting agile methods need to empower the development team and tailor the agile methods and practices to fit the organizational needs.

The literature reveals a large number of studies which have examined the success factors of implementing agile methods in an organization. Previous studies ([5]; [13]; [14]) have investigated the critical success factors in agile development. Indeed, a number of success factors in agile development have been proven as a result of these studies.

Before conducting the factor analysis to study the inter-relations among the success factors of agile development. We reviewed the research on the CSFs of agile software development [15]. As a result, eight success factors were selected as CSFs for agile software projects. The eight success factors are: Delivery strategy, Team capability and training, Agile development techniques, Customer involvement, Project 
management process, Organizational culture, Communication, and Top management support [15].

\section{Data Collection and Preparation}

The survey period lasted 11 weeks to ensure the total response would be suitable for factor analysis. The survey received 131 responses from agile practitioners from more than 28 countries. The appropriateness of conducting factor analysis on the collected data must be checked. Before conducting factor analysis, variables that correlate highly with other variables should be excluded. The same for low correlations [16]. The data set in this study did not contain any variables that are highly correlated or low correlated which need to be excluded prior performing the factor analysis.

The attempts to suggest a minimum sample size for conducting factor analysis have been always debatable. Guadagnoli and Velicer [17] summarized that with small sample size $(<150)$ the researcher should be careful and should only accept the variables with loadings $>0.4$, while larger sample size $(>300)$ should be sufficient for all the loadings values. According to Yong \& Pearce [18], the ratio of participants to variables should be at least 10:1. Since the requirements of conducting factor analysis are met, the factor analysis will be used. The data were analysed using SPSS version 24 .

\section{Factor Extraction}

There are number of factor extraction techniques, such as, principal component analysis (PCA), maximum likelihood, and principal axis factoring (PAF). A principal component analysis (PCA) is used in this study and it was run on an 8-question questionnaire that explore the importance of the CSFs of agile projects. For each success factor a question being asked as a seven-point options from strongly agree to strongly disagree (Likert scale 1-7). The applicability of PCA is assessed prior to the analysis.

The Kaiser-Meyer-Olkin (KMO) is a measure of sampling adequacy ranges from 0 to 1 . According to Williams, Onsman, \& Brown [19], KMO with more than 0.50 should be sufficient for factor analysis. In this study, the KMO using the PCA resulted with 0.820. Kaiser [20] suggests that if the KMO is in the 0.80s, then the factoring of the variables is meritorious. Bartlett's test of Sphericity Sig. should be $<0.05$ to be considered statistically significant, in this study, the Bartlett's test of Sphericity Sig. was $<0.001$.

The PCA disclosed a number of components of which the first two had explained $45.77 \%$ and $12.83 \%$ of the total variance, respectively. The two factors (or components) combined explained $58.61 \%$ of the total variance. Table 1 shows the total eigenvalues and the total variance explained.

Kaiser's criterion is a data extraction technique which retains or extracts all the factors with eigenvalues greater than 1 . This criterion was proposed by Kaiser [21]. In light of this, and following Kaiser's criterion, two components (or factors) were retained (or extracted) for further investigation.

Table 1. Total Eigenvalues and the Total Variance Explained

\begin{tabular}{cccc}
\hline \hline Factor ( or Component) & Total Eigenvalues & \% of Variance & \% Cumulative Variance \\
\hline $\mathbf{1}$ & $\mathbf{3 . 6 6 2}$ & $\mathbf{4 5 . 7 7 7}$ & $\mathbf{4 5 . 7 7 7}$ \\
$\mathbf{2}$ & $\mathbf{1 . 0 2 7}$ & $\mathbf{1 2 . 8 3 5}$ & $\mathbf{5 8 . 6 1 3}$ \\
3 & .862 & 10.777 & 69.390 \\
4 & .640 & 8.001 & 77.391 \\
5 & .611 & 7.641 & 85.032 \\
6 & .489 & 6.111 & 91.143 \\
7 & .423 & 5.291 & 96.434 \\
8 & .285 & 3.566 & 100.000 \\
\hline \hline
\end{tabular}

\section{Factor Interpretation and Labelling}


Having decide the number of factors to be extracted, it is the time to interpret the factors and their loadings. For this purpose, data rotation techniques are used. The aim of factor rotation is to simplify the data structure for better interpretation of the variables. There are two factor rotation techniques, Orthogonal (or Varimax) technique and Oblique (or Oblimin) technique. According to Field [16], Varimax rotation presents the extracted components clearer and easier for interpretation. In this research, Varimax rotation technique is selected and applied on the two extracted components.

Determining the factor loading cut-off and the practical significance level of the factor loadings has been studied and investigated by many research. Field [16] suggests that regardless of the sample size, a factor should be considered reliable if it has four or more loadings with 0.6 or more. On the other hand, Stevens [22] advocates using a factor loading cut off of 0.4 .

Hair et al. [23] provides mapping of the factor loadings and the sample size needed. With factor loading of 0.50 for a sample of 120 and factor loading of 0.45 for a sample of 150 and so on. In light of this, the sample size is 131, so following Hair et al. [23] the minimum factor loading that should be considered practically significant is 0.50 . Table 2 shows the variable loadings on the extracted two components after the rotation and after applying the cut off loading point of 0.50 .

Table 2. The Rotated Variable Loadings after Apply the Cut-off (Factor Loadings $<0.50$ Are Excluded)

\begin{tabular}{lll}
\hline \hline Variable Loading & Component 1 & Component 2 \\
\hline Organizational culture & 0.828 & \\
Customer involvement & 0.791 & \\
Top management support & 0.701 & \\
Team capability and training & 0.626 & \\
Communication & 0.573 & \\
Project management process & & 0.830 \\
The delivery strategy & & 0.663 \\
Agile software development techniques and practices & & 0.578 \\
$\quad$ Total \% of Variance Explained & $\mathbf{4 5 . 7 7 \%}$ & $\mathbf{1 2 . 8 3 \%}$ \\
\hline
\end{tabular}

\section{Discussion}

The eight selected critical success factors of agile software development grouped into two components as shows in Fig. 1.

Factor (or Component) 1: Organizational and People Aspects

Component 1 indicates the importance of organizational aspects and people aspects to the success of agile software development. The highest loading in this component is the organizational culture variable with loading of 0.828 . This indicates that the organizational and people aspects are strongly affected by the organizational culture variable. This also could be interpreted by noticing that the participants of the survey have similar comprehension between the organizational culture and all the organizational and people aspects combined. Thus, people and organizational aspects of the success of agile projects could be understood by the organizational culture more than any other critical success factor. The organizational culture variable was followed by customer involvement variable and top management support with 0.791 and 0.701 loadings, respectively. Team capability variable loaded on component 1 with loading equal to 0.626 .

It is hoped that this could help agile practitioners by noticing that in agile software development projects the organizational culture plays an important role to the people factors as well as to the organizational factors. Success factors like top management support, customer involvement, and team capability proved to be defined and explained by the organizational culture where the agile development project took place more than any other success factor. 
By contrast, this component has low loadings with the technical and project management variables. Project management variable came with 0.02 loading and the delivery strategy variable came with 0.251 loading. This shows the low importance of project management in explaining the organizational and people aspects in agile projects.

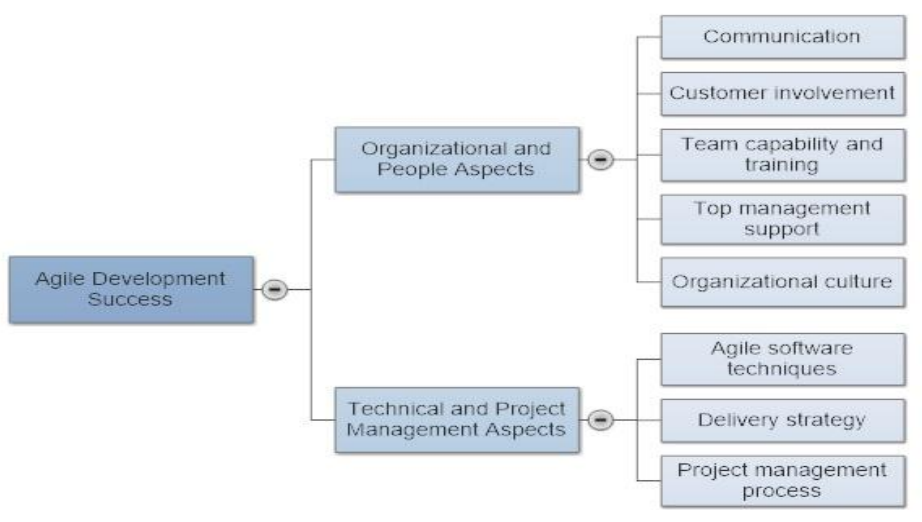

Fig. 1. Critical success factors of agile software development.

Communication variable loaded on component 1 with loading of 0.573 . At the same time communication loaded on component 2 with loading equal to 0.477 (below the chosen cut-off point). This shows that communication plays almost the same role to the variance of organizational and people aspects and the variance of technical and project management aspects. This could be interpreted that in the context of agile software development projects organizational and people aspects influence the way of communication. At the same time technical and project management aspects influence the way the project team communicates during the agile project.

Factor (or Component) 2: Technical and Project Management Aspects

Component 2 shows the importance of project management variable in explaining the variance among the technical aspects in agile projects. The project management variable loaded on component 2 with loading equal to 0.830 . Which suggest that project management variable is highly correlated with the technical facets of agile development. Furthermore, project management variable can be used to describe the technical aspects of agile development project. The delivery strategy variable loaded on factor 2 with 0.663 loading. Agile software development techniques and practices variable loaded with 0.578 loading.

On the other hand, organizational culture variable loaded with -0.477. Same for customer involvement, top management support, and team capability and training variables all were loaded with negative values. This suggest that all these variables do not play a role in explaining the variance in the technical and project management aspects. Moreover, the negative loadings suggest that these variables explain the variance in the other factor.

The technical aspects in agile software development are influenced by the project management approach more than any other success factors. This should help agile practitioners when they select the project management approach for agile projects. The selected project management approach will influence the technical aspects of agile projects such as delivery strategy.

\section{Conclusion and Future Work}


In this paper, the relationships among the critical success factors of agile software development is explored using factor analysis. PCA is used and resulted with grouping the success factors into two components. The first component concerning about people and organizational related success factors. While the second component concerning about technical and project related success factors.

Organizational culture success factor was the highest loading on the component of people and organizational aspects. This shows that the organizational culture is the most success factor that could explain the people and organizational aspect in the context of agile development projects. On the other hand, project management process success factor revealed to be the highest loading on the component of the technical and project management aspects. This indicate that project management process success factor influences the technical factors in the success of agile development projects.

The future work of this study could be doing the survey with more responses which will give the factor analysis more validity. Future work also may focus on one or more of the success factors and to study the inter-relation among them and how they influence the success in agile projects. More could be explored on each of the resulted components and how the inter-relationships between the factors could be understood for achieving the success in the context of agile development projects. The analysis can be extended to develop an instrument for predicting agile success. Alternatively, it could be used as a diagnostic to indicate causes of weakness which should be addressed with appropriate training.

\section{References}

[1] Beck, K., Mike, B., Arie, V. B., \& Alistair, C. (2001). Ward cunningham, martin fowler, james grenning. Manifesto for Agile Software Development.

[2] Dingsøyr, T., Nerur, S., Balijepally, V. G., \& Moe, N. B. (2012). A decade of agile methodologies: Towards explaining agile software development. Journal of Systems and Software, 85(6), 1213-1221.

[3] Highsmith, J., \& Cockburn, A. (2001). Agile software development: The business of innovation. Computer, 34(9), 120-122.

[4] Leidecker, J. K., \& Bruno, A. V. (1984). Identifying and using critical success factors. Long Range Planning, 17(1), 23-32.

[5] Chow, T., \& Cao, D. B. (2008). A survey study of critical success factors in agile software projects. Journal of Systems and Software, 81(6), 961-971.

[6] Dyba, T., \& Dingsoyr, T. (2009). What do we know about agile software development? IEEE Software, 26(5), 6-9.

[7] Vijayasarathy, L., \& Turk, D. (2008). Agile software development: A survey of early adopters. Journal of Information Technology Management, 19(2), 1-8.

[8] Pelrine, J. (2011). On understanding software agility - A social complexity point of view. CognitiveEdge.Com, 13, 26-37.

[9] Agile state report. (2016). 10th Annual State of Agile Report, State of Agile Report 2016, Retrieved September 06, from https://explore.versionone.com/state-of-agile/versionone-10th-annual-state-ofagile-report-2

[10] Abrahamsson, P., Salo, O., Ronkainen, J., \& Warsta, J. (2002). Agile software development methods: Review and analysis.VTT Technical report.

[11] Turk, D., France, R., \& Rumpe, B. (2014). Assumptions underlying agile software development processes. Journal of Database Management, 16(4), 62-87.

[12] Pikkarainen, M., Salo, O., Kuusela, R., \& Abrahamsson, P. (2012). Strengths and barriers behind the successful agile deployment-insights from the three software intensive companies in Finland. Empirical Software Engineering, 17(6), 675-702. 
[13] Stelzmann, E., Kreiner, C., Spork, G., Messnarz, R., \& Koenig, F. (2010). Agility meets systems engineering: a catalogue of success factors from industry practice. Proceedings of the European Conference on Software Process Improvement, 245-256.

[14] Misra, S. C., Kumar, V., \& Kumar, U. (2009). Identifying some important success factors in adopting agile software development practices. Journal of Systems and Software, 82(11), 1869-1890.

[15] Aldahmash, A., Gravell, A. M., \& Howard, Y. (2017). A review on the critical success factors of agile software development. systems, software and services process improvement. Proceedings of the 24th European Conference, EuroSPI 2017.

[16] Field, A. (2009). Discovering Statistics Using SPSS. Sage publications.

[17] Guadagnoli, E., \& Velicer, W. F. (1988). Relation to sample size to the stability of component patterns. Psychological bulletin, 103(2), 265.

[18] Yong, A. G., \& Pearce, S. (2013). A beginner's guide to factor analysis: Focusing on exploratory factor analysis. Tutorials in Quantitative Methods for Psychology, 9(2), 79-94.

[19] Williams, B., \& Onsman, A., \& Brown, T. (2010). Exploratory factor analysis: A five-step guide for novices. Australasian Journal of Paramedicine, 8(3), 1-13.

[20] Kaiser, H. F. (1974). An index of factorial simplicity. Psychometrika, 39(1), 31-36.

[21] Kaiser, H. F. (1960). The application of electronic computers to factor analysis. Educational and psychological measurement, 20(1), 141-151.

[22] Stevens, J. P. (2012). Applied multivariate statistics for the social sciences. Routledge.

[23] Hair, J. F., et al. (2009). Multivariate data analysis: A global perspective.

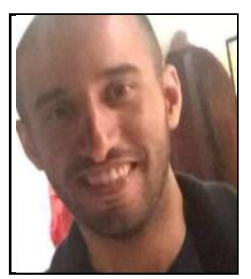

Abdullah Aldahmash is a PhD student at the School of Electronics and Computer Science, University of Southampton. He completed his master degree in information systems at King Saud University. His current research is about agile software development and empirical assessment of software development. His current research interests include software metrics, secure systems, and enterprise systems.

Andrew Gravell (aka Andy Gravell) studied maths and computer science at Cambridge. Worked as a software developer for 7 years, mostly at IBM's hursley laboratory, working on a range of projects writing software for microprocessor-based systems. In 1987 joined ECS, Southampton, as a lecturer in computer science, promoted to senior lecturer / associate professor in 2002. His research interests include: public understanding of computer science; computer science education; and the computer science pipeline; empirical approaches to software development; agile methods; and lean information technology.

Yvonne Howard is a senior research fellow of Computer and Web Science at the University of Southampton. She is a member of the Web and Internet Science Group. Yvonne is the manager of the rapid Innovation and Research Team for the University's Centre for Innovation in Technology and Education (CITE). Her research interests include agile and social methods of user participation in the development process. 